\title{
Alcohol y atención primaria de salud: el difícil camino hacia la normalización
}

\author{
Antoni Gual*; Joan Colom**. \\ * Jefe de la Unidad de Alcohologia de la Generalitat. ICPP. IDIBAPS. Hospital Clínic de Barcelona \\ * Director dela Unidad de Drogodependencias y Sida. Departamento de Sanidad. Generalitat de Catalunya. \\ Enviar correspondencia a: \\ Dr. Antoni Gual. Cap de la Unitat d'Alcohologia de la Generalitat. Institut de Psiquiatria i Psicologia. IDIBAPS. \\ Hospital Clínic. Mejia Lequerica, s/n · 08010 Barcelona. SPAIN .TF. +34932279923 · e-mail: tgual@clinic.ub.es
}

\section{RESUMEN:}

El consumo de alcohol y los problemas relacionados con dicho consumo se distribuyen a lo largo de un continuum que empieza con el consumo ocasional y termina en la dependencia severa. Frente a ello, la administración sanitaria debe garantizar una continuidad asistencial que se inicia en la educación para la salud y la identificación precoz de los bebedores de riesgo y termina en los centros de larga estancia para dependientes con escasas probabilidades de recuperación.

A la atención primaria de salud le corresponde asumir la identificación precoz de los bebedores de riesgo, así como las intervenciones breves para reducir los consumos y la derivación de los casos mas severos a los centros especializados. Para garantizar el buen funcionamiento del continuum asistencial es precisa una mejor coordinación entre la atención primaria y la especializada, así como una formación específica de los equipos de atención primaria. Se postula que para promover estos cambios es imprescindible ofrecer formación específica en el marco de los espacios de formación continuada, siendo una opción consistente la participación de los especialistas en adicciones ubicados en el mismo ámbito geográfico, con quienes ulteriormente deberá mantenerse la coordinación asistencial. Asimismo, se plantea la necesidad de incentivar las intervenciones preventivas mediante fórmulas contractuales que en la actualidad ya son utilizadas por algunas administraciones sanitarias, aunque todavía no se hayan evaluado.

Palabras clave: Alcohol, Atención Primaria de Salud, Consumo de riesgo.

\section{SUMMARY:}

Alcohol consumption and alcohol related problems expand through a continuum that ranges from occasional drinking to severe dependence. According to that, Health authorities must guarantee a continuity of care, that starts with health education and early identification of risky drinkers, and finishes at residential centers for severely impaired alcoholics.

Primary Health Care Centers should focus on early identification of risky drinkers, delivery of brief interventions to reduce alcohol consumption, and appropriate referral of severe cases to specialized centers. Two key points seem to be crucial to ensure continuity of care: appropriate training of Primary Health Care professionals, and good coordination between Primary Health and specialized services. Training is feasible when integrated in the regular CME hours already existing in Primary Health Care Centers. Alcohol specialists with whom coordination will be established later on, may also act as trainers.

Finally, it is stated that preventive activities in Primary Health Care should be recognized and incentivated through contractual measures. Some health authorities have already started to use this strategy but they have not been evaluated yet..

Key words: Alcohol, Primary Health Care, Hazardous drinking, risky drinking.

\section{INTRODUCCIÓN}

D Desde una perspectiva de salud pública existen con relación a las bebidas alcohólicas por lo menos dos verdades incuestionables: una, el alcohol es una de las principales causas de enfermedades prevenibles; y dos, a pesar de ello los recursos que se destinan para prevenirlas siguen siendo más que insuficientes.

Es obvio que un problema de salud pública exige no sólo una respuesta asistencial coherente e integral, sino 
también medidas legislativas en ámbitos tan esenciales como la fiscalidad, publicidad, disponibilidad, etc.

Sin pretender en absoluto restar importancia a este tipo de medidas, imprescindibles pero impopulares y difíciles de asumir por tanto para los políticos, nos centraremos en este editorial en la respuesta asistencial de la sanidad pública española frente a las consecuencias negativas del consumo de bebidas alcohólicas.

Al igual que con otras patologías, se han propuesto niveles de intervención distintos en función del tipo y severidad de los problemas detectados. De hecho, los problemas relacionados con el alcohol se mueven en un continuum de severidad creciente que, utilizando terminología OMS, empieza en los consumos de riesgo, y termina en la dependencia alcohólica. Es obvio que la respuesta asistencial debe ser coherente e integral, de modo que frente al continuum de los problemas relacionados con el alcohol debe proponerse una oferta asistencial que garantice la continuidad en la atención sanitaria a dichos problemas.

Pero la organización de esta respuesta asistencial es mucho más compleja de lo que a simple vista pueda parecer. En efecto, a la hora de planificar una intervención hay diversos parámetros a tener en cuenta; entre ellos destacaremos los siguientes: prevalencia del problema, severidad del mismo y complejidad de las intervenciones necesarias. Cuanto más prevalente sea la problemática y menos compleja sea la intervención, mayor será el protagonismo que deba otorgarse a los dispositivos de atención primaria'. Contrariamente, intervenciones complejas en patologías poco frecuentes deberán ubicarse en el terreno de los dispositivos especializados.

En el ámbito del Estado español probablemente pueda afirmarse que se ha recorrido un largo camino, pero que aún es largo el que queda por recorrer. Quizás el mayor logro sea la normalización del tratamiento de pacientes con dependencia alcohólica en centros especializados. Así, lo que en los años 70 fueron experiencias pioneras (Dispensarios Antialcohólicos del PANAP) se ha convertido, también gracias a la epidemia de la heroína, en una red asistencial pública modélica en Europa, con matices regionales en los que aquí no vamos a entrar.

Desde esa misma óptica positiva, los últimos decenios han visto el desarrollo de unidades hospitalarias de desintoxicación, capaces de dar una respuesta eficaz a las necesidades de hospitalización de corta estancia para desintoxicación alcohólica. La perspectiva en cambio es menos halagüeña cuando evaluamos los dispositivos asistenciales de larga estancia, donde la oferta existente es claramente inferior a la necesaria, y a menudo con dotaciones presupuestarias que impiden una correcta profesionalización de equipamientos como comunidades terapéuticas, pisos protegidos, talleres ocupacionales, etc.

En el otro extremo del continuum, tampoco tenemos muchos motivos de satisfacción. En el ámbito de la atención primaria de salud se han desarrollado pocos programas formativos, y en su inmensa mayoría se han orientado hacia el tratamiento del alcoholismo, y no hacia la identificación y abordaje de los bebedores de riesgo, que sin duda debiera ser la tarea prioritaria en atención primaria.

Al hablar de la atención primaria se plantean necesariamente tres cuestiones: que nivel de intervención deben tener los profesionales de atención primaria en los problemas relacionados con el alcohol, que formación deben recibir para realizar dichas intervenciones, y finalmente que recursos deben serles facilitados para que realicen eficientemente sus funciones.

En la actualidad existe un amplio consenso a la hora de responder a la primera de dichas cuestiones: corresponde a la atención primaria la identificación precoz de los consumos de riesgo y de los problemas relacionados con el alcohol, aunque existe menos unanimidad a la hora de plantear si dicha identificación precoz debe realizarse mediante técnicas de cribado ${ }^{2}$ u oportunísticamente ${ }^{3}$. En esa población las técnicas de consejo breve han demostrado una notable eficacia ${ }^{4,5}$, y una óptima relación coste-eficacia ${ }^{6,7}$, y sin duda deben ser conocidas y utilizadas por los profesionales de atención primaria.

También existe acuerdo en contemplar la atención de los pacientes alcohólicos como responsabilidad de la atención especializada. Correspondería aquí a la atención primaria la labor de derivación, que exige consensuar criterios, y por defecto acaba implicando que los casos menos severos de dependencia alcohólica puedan iniciar tratamiento en la propia atención primaria.

Los profesionales de la atención primaria deben por tanto disponer de una formación en técnicas de identificación precoz e intervención breve, así como conocimientos básicos sobre el tratamiento de la dependencia alcohólica y los criterios de derivación a los centros especializados. Es obvio que la coordinación con dichos centros constituirá una piedra angular para garantizar el continuum asistencial al que antes hacíamos referencia.

Aunque este sea un planteamiento correcto, a ningún observador experto escapa su implementación topa con muchos obstáculos. Existen en la atención primaria dificultades objetivas para la integración de los programas de alcohol en las rutinas asistenciales ${ }^{8}$, al igual que existen dificultades estructurales para facilitar la coordinación entre los centros especializados y la atención primaria de salud. En este último apartado, la integración de dichos centros en la estructura sanitaria general ha supuesto sin duda un gran avance en aque- 
llas comunidades autónomas donde dicha integración ya se ha consumado.

Dos factores clave para facilitar la integración de las técnicas de identificación precoz e intervención breve en bebedores de riesgo de forma satisfactoria en la práctica clínica cotidiana de los equipos de atención primaria son la formación y el soporte institucional. Existe evidencia de que la formación es bien recibida cuando procede de los especialistas que trabajan en el mismo ámbito geográfico, y se realiza en el marco de los espacios ya existentes de formación médica continuada. Asimismo, los nuevos sistemas de contratación de servicios ofrecen a la administración sanitaria la posibilidad de reconocer e incentivar las actividades de promoción de la salud, aunque nos hallemos todavía en los albores de esta nueva filosofía de gestión asistencial.

Sin duda alguna, conseguir que la atención primaria de salud aborde de forma sistemática el consumo de alcohol de los pacientes es una tarea difícil pero necesaria. Para conseguirlo hace falta dotar a los profesionales de formación, mayor disponibilidad de tiempo y una mejor coordinación con los centros específicos. La OMS calcula que la implementación de programas de alcohol en el 50\% la atención primaria de salud puede representar anualmente en Europa una disminución de 778.000 años de vida ajustados por discapacidad (DALYs), más que ninguna otra política preventiva. Son cifras más que elocuentes para justificar un cambio en las prioridades de las administraciones sanitarias, sobretodo si a los costes económicos añadimos las repercusiones sociales que inevitablemente comportan los problemas relacionados con el alcohol.

\section{REFERENCIAS}

1 Gual A, Colom J. Papel de la atención primaria de salud en los problemas derivados del consumo de bebidas alcohólicas Med Clin (Barc). 2001 Feb 3;116(4):136-7

2 Heather N, Anderson P, Gual A, Seppa K. Some screening is necessary to identify excessive drinkers early in primary care. BMJ. 2003 Mar 8;326(7388):550

3 Beich A, Thorsen T, Rollnick S. Screening in brief intervention trials targeting excessive drinkers in general practice: systematic review and meta-analysis. BMJ. 2003 Sep 6;327(7414):536-42.

4 Moyer A, Finney JW, Swearingen CE, Vergun P. Brief interventions for alcohol problems: a meta-analytic review of controlled investigations in treatment-seeking and non-treatment-seeking populations. Addiction 2002;97:279-92.

5 Poikolainen K. Effectiveness of brief interventions to reduce alcohol intake in primary health care populations: a meta-analysis. Prev Med 1999;28:503-9.

6 Wützke SE, Shiell A, Gomel MK, Conigrave KM. Cost effectiveness of brief intervention for reducing alcohol consumption. Social Science and Medicine 2001;52:863-870.

7 Fleming MF, Mundt MP, French MT, Manwell LB, Stauffacher EA, Barry KL. Benefit-cost analysis of brief physician advice with problem drinkers in primary care settings. Medical Care 2000;38:7-18.

8 McAvoy BR, Kaner EF, Lock CA, Heather N, Gilvarry E. Our Healthier Nation: are general practitioners willing and able to deliver? A survey of attitudes to and involvement in health promotion and lifestyle counselling. $\mathrm{Br}$ J Gen Pract. 1999 Mar;49(440):187-90 
